\title{
Raman Spectral Data De-noising based on Wavelet Analysis
}

\author{
Nitendra Kumar \\ Department of Mathematics \\ School of Engg. \& Tech. \\ Sharda University Greater \\ Noida 201310, India
}

\author{
A. H. Siddiqi \\ Prof. Department of \\ Mathematics \\ School of Engg. \& Tech. \\ Sharda University Greater \\ Noida 201310, India
}

\author{
Khursheed Alam, PhD \\ Department of Mathematics \\ School of Engg. \& Tech. \\ Sharda University Greater \\ Noida 201310, India
}

\begin{abstract}
Nowadays, most analytical instruments in modern laboratories are computerized, partly owing to the rapid development of advanced micro-electronic technology. Digitalized spectroscopic data can be exported from these instruments very easily for subsequent signal processing. Raman Spectroscopy is widely recognized as powerful, nondestructive techniques for characterizing materials. The key to realize the qualitative and quantitative analysis is data processing and analysis. But signals in Raman spectral analysis often have noise, which greatly influences the achievement of accurate analytical results. The de-noising of Raman spectral is an important part of de-noising. Wavelet functions are localized both time and frequency (or scale) and in time, via dilations and translations of the mother wavelet, respectively, both time and frequency information are maintained after transformation. This paper presents wavelet based de-noising method for Raman Spectral data of $\mathrm{Sr}^{2+}$ modified PMN-PZT and compared the results with Daubechies, Coiflet, Symlet.
\end{abstract}

Keywords Raman Spectral Signal, Wavelet Analysis, Signal De-noising

\section{INTRODUCTION}

Nowadays, most of the analytical instruments in modern laboratories are computerized, particularly owing to the rapid development of micro-electronics technology. Digital spectroscopic data can be explored from these instruments very easily for subsequent signal processing. Raman spectroscopy is advantageous because quick and accurate measurements can often be made with destroying the sample and with minimal sample preparation.

Raman spectroscopy (RS, named after C. V. Raman) is a kind of molecule scattering spectroscopy, which is characterized by the frequency excursion that can show the information of molecule. Raman Spectroscopy is used to retrieve the vibrational information of molecules. Vibrational spectroscopic features arise from the loss or gain of energy by photons which are inelastically scattered from a vibrating sample. This energy change results in an alteration of the photon's wavelength which in the case of materials is directly related to the vibrational states of its properties. Therefore, quantitative biochemical information can be extracted from the spectra [2].

The Raman effect is observed when a very small number of photons incident on a molecule are inelastically scattered, that is, scattered at different frequencies than the frequency of the incident photons.
The weak nature of Raman effect results in spectra with very low signal-to-noise ratio (SNR). The noise in Raman spectra takes one of these forms: high frequency noise, low-frequency noise and cosmic spikes. High frequency noise comes from the acquisition electronics and other sources of system variation, low frequency noise arise from ambient light entering the spectrograph and fluorescence emission from the sample and cosmic spikes are spurious, very narrow, spikes appearing in the Raman spectra. They are an artifact of the detection electronics [2].

A Raman spectrum is a plot of the intensity of scattered light versus the energy difference between the incident photons and the Raman-scattered photons, and contains information about the chemical composition and the molecular structure of a substance. A major limitation of Raman spectroscopy is the weakness of the Raman effect which results in very low signals.

\section{BASIC PRINCIPLE OF WAVELET TRANSFORM}

Wavelet transforms is a great tool for de-noising signals. In fact, it is a series of different frequencies block signals which are de-composed by their respective resolutions of original spectral. The frequency of each point in original spectrum is obvious. The flexible window is provided by emergence of wavelet analysis.

If $\varphi(t) \in \mathrm{L}^{2}(\mathrm{R})\left(\mathrm{L}^{2}(\mathrm{R})\right.$ is a real space of square integrable), its Fourier transform is $\varphi(\omega)$, when contents the admissible condition:

$$
\int_{-\infty}^{\infty} \varphi(t) d t=0
$$

$\varphi(t)$ is called a basic wavelet or generating wavelet. The continuous wavelet is obtained by generating function $\varphi(t)$ after expansion and translation:

$\varphi_{\mathrm{a}, \mathrm{b}}(\mathrm{t})=\frac{1}{\sqrt{|\mathrm{a}|}} \varphi\left(\frac{\mathrm{t}-\mathrm{b}}{\mathrm{a}}\right) ; \mathrm{a}, \mathrm{b} \in \mathrm{R}, \mathrm{a} \neq 0$

Equation (2) is called a wavelet sequence. Where a is called the extension scale, when a increases, the local property weaken, the local property enhances. The overall properties enhance. When a decreases, the local property weaken; $\mathrm{b}$ is 
called the shift operator. $\varphi\left(\frac{\mathrm{t}-\mathrm{b}}{\mathrm{a}}\right)$ is recorded as $\varphi_{\mathrm{a}, \mathrm{t}}$, it is a wavelet function which is flexed by a, translated by $b$ of the generating function $\varphi(t)$.

The wavelet transform of $\varphi(t)$ is

$\mathrm{W}_{\mathrm{f}}(\mathrm{a}, \mathrm{b})=|\mathrm{a}|^{1 / 2} \int_{-\infty}^{\infty} \varphi(\mathrm{t}) \psi\left(\frac{\mathrm{t}-\mathrm{b}}{\mathrm{a}}\right) \mathrm{dt}$

If the Fourier transform $\psi(\omega)$ of function $\psi(t)$ meets the admissible conditions:

$\mathrm{C}_{\psi}=\int_{-\infty}^{\infty} \frac{|\psi(\omega)|^{2}}{|\omega|} \mathrm{d} \omega<\infty$

So wavelet transform is reversible, its inverse is:

$\psi(\mathrm{t})=\frac{1}{\mathrm{C}_{\psi}} \int_{-\infty}^{\infty} \int_{-\infty}^{\infty} \frac{1}{\mathrm{a}^{2}} \mathrm{~W}_{\mathrm{f}}(\mathrm{a}, \mathrm{b}) \psi_{(\mathrm{a}, \mathrm{b})}(\mathrm{t}) \mathrm{dadb}$

\subsection{Raman Spectroscopy and Wavelet Transform}

Wavelets are functions that are localized in both the time and frequency domains by the using of wavelet transform, one can effectively extract both time and frequency information from a time-varying signal, which makes it suitable for nonperiodic signals. In the case of Raman spectral signal, Wavelet transform (Discrete Wavelet Transform) can be very useful because it determines the actual position (Raman Shift) of these frequencies in the spectrum instead of the time. For de-noising of Raman spectral signal, DWT decomposes the spectrum into selected contributions (detailed coefficients or approximations) labeled by changes in the scale and translation parameters. Each contribution at a different scale (resolution level) gives information about the different frequencies and the positions of these frequencies in the original spectrum. The approximation carries out the information about the samples, whereas the detailed coefficients carries the identity of the Raman spectral signal and the undesired high-frequency shot-noise and it very useful for detecting spectral features and filtered these features apart from the noise $[1,2]$.

The motivation to use wavelet as a possible alternative is to discover new ways to reduce computational complexity and to achieve better noise reduction performance. The wavelet denoising technique is called thresholding. It is divided in three steps. The first one consists of computing the coefficients of the wavelet transform (WT) which is a linear operation. The second one consists of thresholding these coefficients. The last step is the inversion of the thresholded coefficients by applying the inverse wavelet transform, which leads to the denoised signal. This technique is simple and efficient. In this paper we were de-noise the Raman Spectral signal of PZTPMN using Daubechies, Symlets and Coiflet wavelet at different levels $[3,5,6,7,8,9]$.

\section{TEST ANALYSIS AND EVALUATION}

\subsection{Signal - to - noise ratio (SNR)}

In order to verify the performance of the proposed denoising approach, computer generated noises with variable amplitudes are added to well-known benchmark signals; moreover, the classical algorithms are performed for comparison. A number of quantitative parameters can be used to evaluate the performance of the de-noising procedure in terms of the reconstructed signal quality. In this case, the following parameters are compared:

$$
\operatorname{SNR}(\mathrm{db})=10 \ln \frac{\sum_{\mathrm{k}=1}^{\mathrm{N}} \mathrm{x}^{2}(\mathrm{k})}{\sum_{\mathrm{k}=1}^{\mathrm{N}}\left[\mathrm{x}(\mathrm{k})-\mathrm{x}^{\prime}(\mathrm{k})\right]^{2}}
$$

Where $\mathrm{X}^{\prime}(\mathrm{k})$ is the de-noise signal, and $\mathrm{X}(\mathrm{k})$ is the original signal. the constant, $\mathrm{N}$, is the number of sample composing the signals.

\subsection{Root-mean square error (RMS)}

$\mathrm{RMSE}=\sqrt{\frac{\sum_{\mathrm{k}=1}^{\mathrm{N}}\left[\mathrm{x}(\mathrm{k})-\mathrm{x}^{\prime}(\mathrm{k})\right]^{2}}{\mathrm{~N}}}$

\section{SIMULATION SETTING}

Raman Spectral data of $\mathrm{Sr}^{+2}$ modified PZT-PMN (with $\mathrm{Sr}=$ 0.025 ) has been collected by Raman spectrometer of Remisaw $\mathrm{Rm}-1000$ spectroscope, which use $1.19 \mathrm{~nm}$ as a unit range of $200-1000 \mathrm{~cm}^{-1}$. The experiment was carried at a room temperature about $25^{\circ} \mathrm{C}$. The data has been obtain in .txt format and has two kinds of $876 \times 2$ data that represents the intensity-Raman shift and the intensity-pixel. Only the intensity value (876X1) has been exported as one dimensional.

In case of Raman Spectrum, which is constituted of equally spaced points with intensity dependent on the positions of such points, and reasonable symmetry of the bands, Wavelet based (Daubechie's, Symlet's and Coiflet) were compared in order to ensure the symmetry. Matlab 2010a is as a platform in this experiment, the spectral de-noising simulation is done by the wavelet toolbox. The choice of decomposition level $n$ depends on the selected wavelet family, signal sampling, and spectral resolution, in order to obtain spectral decomposition. The decomposition of the signal was performed using Db5, Sym5 and coif5 with 3 levels.

The original spectrum in figure 1 and de-noise spectrum in figure 2, 34 respectively, can be compared, de-noised spectrum is more smoothly, some random burr weak signals of original measurement spectra disappeared after wavelet transformation. The center wavelength position and intensity of de-noised spectrum is identical with original spectrum. The original signals of some low strength noise also had good effect in noise filtering.

\section{CONCLUSION}

The method of wavelet transform for Raman spectral data of PZT-PMN is studied in this paper. The data de-noising methods which based on wavelet transform using db5, sym5 and coif5 with level 3 is give better result. the signal-to-noise ratio and root-mean square error shows that the de-noised results improve the background of original result. 


\section{REFERENCES}

[1] Chen. S., Lin X., Yuen C., Padmanabhan S, Beuerman Roger W. and Liu Q, 2014, Recovery of Raman Spectra with low signal-to-noise ratio using Wiener estimation, Optical Society of America. Vol. 22. No. 10.

[2] Raman C. V., 1928, "A new type of secondary radiation", Nature, 121, 619.

[3] Yang Y, Guo L. and Li F., 2011, “Application of wavelet transform in spectral data de-noising", 978-1-0246-4/11, IEEE.

[4] Gang F., 2008, "Noise removal of Raman spectra using interval thresholding method", DOI 0.1109/IITA, 573, IEEE.

[5] Chen C., Peng F., Cheng Q and Xu D., 2009, "Application of Wavelet Packet Transform to Compressing Raman Spectra Data", Proc. of SPIE Vol. $7280728009-1$.

[6] Ehrentreich F., Summchen F, 2001, "Spike removal and De-noising of Raman spectra by wavelet transform methods", Analytical chemistry, Vol. 73, no. 17, pp. 4364-4373.

[7] Cai W., Wang L., Pan Z, Zuo Z., Xu C., Shao, 2001, "Application of the Wavelet of Raman spectra", Journal of Raman Spectroscopy, Vol. 32, No. 3, pp. 207-209.

[8] Silveria Jr. L., Benito B.,. Amaro Z. R and Tadeu T. P. M., 2010, "Discrete wavelet transform for de-nosing Raman spectra of human skin tissue used in a discriminate diagnostic algorithm", Journal of Instrumentation science \& Technology, 38:268-282, Taylor \& Francis, ISSN: 1073-9149.

[9] Ramos, P. M, Ruisa'nchez I., 2005, "Noise and background removal in Raman spectra of ancient pigments using wavelet transform", J. Raman Spectroscopy, 36(9), 848-856.

[10] Hu Y., Jiang T., Shen A., Li W., Wang X., and Hu J., 2007, "A background elimination method based on wavelet transform for Raman spectra", Chemometer Intell. Lab. Syst, 85(1), 94-101.

[11] Kumar A. and Mishra S. K., 2014, Effects of Sr2+ substitution on structural, dielectric and piezoelectric properties of PZT-PMN ceramic. Int. J. Miner. Metall. Mater, (Feb. 2014), Vol. 21, No. 2.

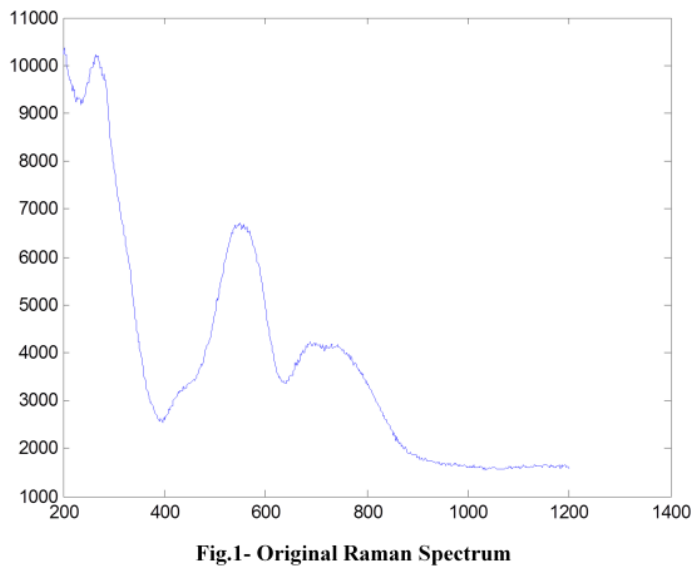

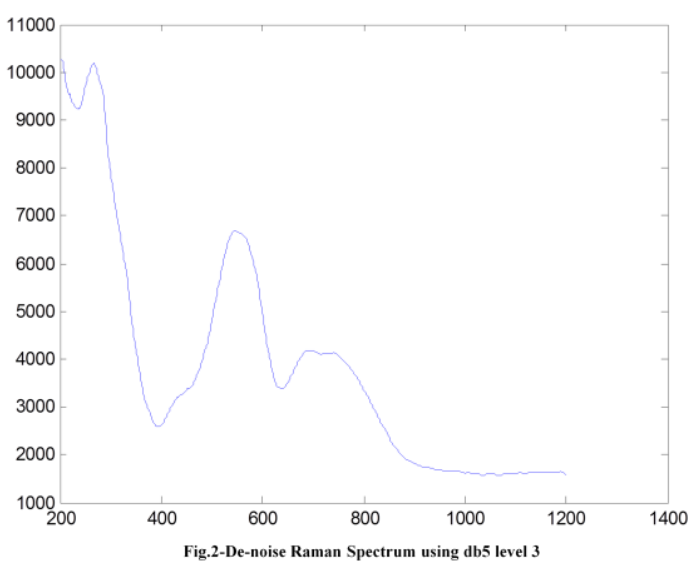
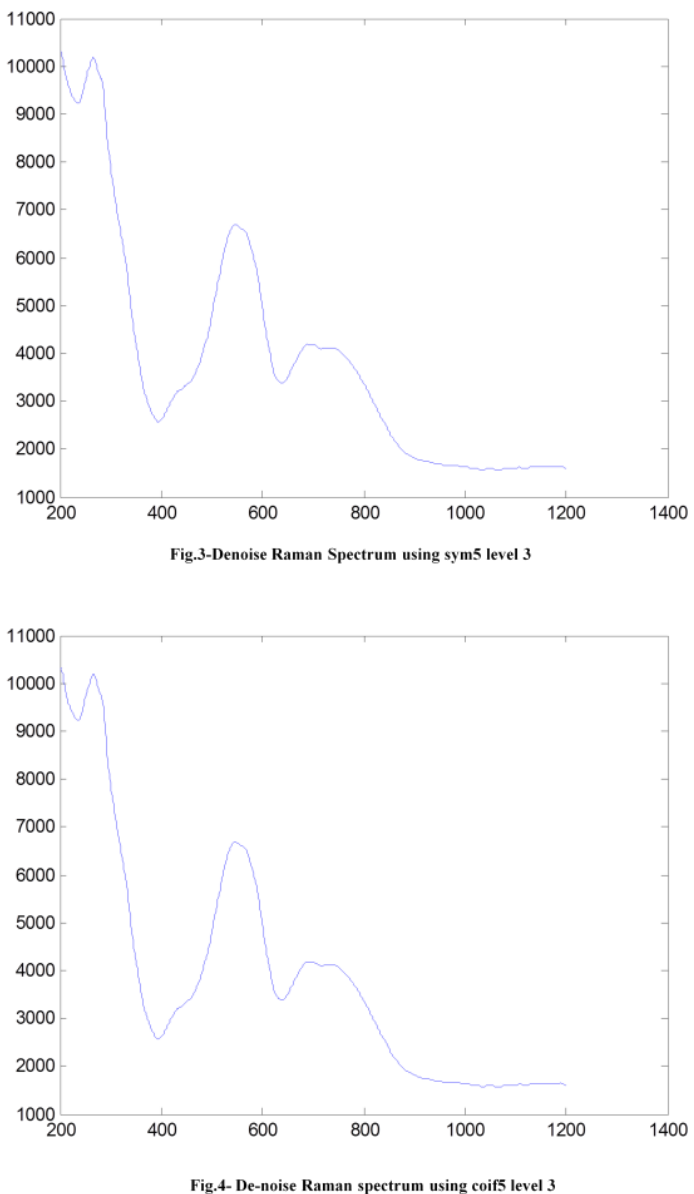

\begin{tabular}{|l|l|l|}
\hline Wavelet Names & SNR & RMSE \\
\hline Db5 & 40.4109 & 28.4313 \\
\hline Sym5 & 40.2276 & 29.0334 \\
\hline Coif5 & 40.3205 & 28.7259 \\
\hline
\end{tabular}

Table 1. 